INRA Prod. Anim., 2000, 13 (5), 287-301

\section{S. BONNY}

INRA-INAPG, UMR Economie publique, BP 1, 78850 Grignon

courrie : bonny@grignon.inra.fr
Les consommateurs, l'agriculture, la qualité et la sécurité des aliments : une analyse du questionnement des consommateurs et des réponses apportées.

La sécurité sanitaire des aliments est devenue depuis quelque temps un objet de vives préoccupations en France avec les problèmes de maladie de la vache folle, de listériose, d'OGM, de dioxine, etc. De fortes critiques s'expriment envers l'agriculture et l'alimentation modernes. L'amélioration de la sûreté et de la qualité de l'alimentation est ainsi devenue un objectif très souvent mis en avant par de nombreux acteurs de la filière. Dans ce contexte, l'article ne vise pas à faire une analyse d'une crise particulière ou d'un épisode de forte crise comme celui lié à l'E SB fin 2000. II cherche au contraire une vue d'ensemble à plus long terme et une approche de synthèse sur diverses questions sous-jacentes en jeu : opinions des consommateurs en matière de qualité et de sûreté de l'alimentation, facteurs à l'origine des remises en cause actuelles, adéquation des réponses proposées.

«Toute la structure sociale est fondée sur la croyance ou sur la confiance» Paul Valéry

Actuellement, dans I'Union Européenne et en particulier en France, de fortes critiques se font entendre envers l'agriculture moderne

\section{Résumé}

L'article analyse les questionnements et les critiques souvent formulées aujourd'hui envers l'agriculture et l'alimentation modernes, et les réponses apportées par les acteurs concernés. Dans un premier temps, on cherche à mieux connaître la perception des risques par les consommateurs en matière de qualité et de sûreté des produits alimentaires en s'appuyant notamment sur diverses enquêtes récentes. L'article présente ensuite divers facteurs explicatifs du questionnement envers le type d'agriculture et d'alimentation actuel. A partir de là, on examine si les solutions proposées aux risques dénoncés et à la crise de confiance semblent adaptées. Or celles-ci apparaissent souvent comme des réponses essentiellement techniques à une demande ou des préoccupations qui ne sont pas seulement d'ordre technique. accusée d'effets négatifs, notamment en matière de qualité et de sécurité sanitaire des produits et d'environnement. Des voix de plus en plus nombreuses, entre autres dans les médias et dans divers mouvements associatifs, dénoncent les méfaits du productivisme. Cependant, dans le même temps, si on mesure l'incidence effective des risques alimentaires sur la santé, celle-ci paraît très faible (Apfelbaum 1998, Tubiana 1998, Nizard 1999, Tubiana et al 1999) : l'espérance de vie s'allonge, les décès ou maladies graves imputables à l'alimentation proviennent le plus souvent des comportements et des choix des consommateurs eux-mêmes (notamment d'une forte consommation de tabac, d'alcool, etc.) et non de la sûreté des produits (tableau 1). 
Tableau 1. Nombre de décès selon diverses causes en 1996 en France (source : Nizard 1999 et données INSERM).

NB : La marge d'erreur est importante et toutes les causes ne sont pas listées.

\begin{tabular}{|lr|}
\hline & \multicolumn{2}{c|}{ Total } \\
Maladies cardio-vasculaires & 173269 \\
\hline Cancers, excepté ceux d'origine tabagique et alcoolique & 81952 \\
\hline Cancers liés à la consommation de tabac & 53731 \\
\hline Circulation routière & 26170 \\
- dont polluti on atmosphéri que & 17629 \\
- dont acci dents de la route & 8541 \\
\hline Consommation d'alcool & 13855 \\
- dont cancers d'ori gi ne alcooli que très probable & 12082 \\
- dont maladi es du foi e d'ori gi ne alcooli que & 8961 \\
- dont dépendance et psychose alcooli ques & 2941 \\
\hline Suicides & \multicolumn{2}{|c|}{11279} \\
\hline Infections alimentaires et/ou intestinales & 625 \\
- dont toxi -i nfections alimentai res & 100 \\
- dont li stéri ose & 25 \\
- dont infections i ntesti nales (di verses causes) & 500 \\
\hline Total des décès toutes causes & \multicolumn{2}{|c|}{$5 \mathbf{7 7 5} \mathbf{7 7 5}$} \\
\hline
\end{tabular}

L'état de l'environnement de son côté n'a pas connu que des dégradations, il s'est amélioré depuis un siècle en Europe en certains domaines comme l'état bactériologique des eaux, le smog urbain, le reboisement de zones de montagne naguère très érodées suite à leur déforestation, etc. Mais les questionnements concernant les liens entre agriculture moderne, environnement, alimentation et santé sont importants et se retrouvent dans de nombreux pays ; à l'échelle planétaire ils renvoient à la question cruciale de la durabilité des modèles de production agricole et des modèles alimentaires actuels en liaison avec l'accroissement de la population prévu pour les prochaines décennies.

Ce texte propose une analyse de ces questionnements et des réponses apportées par les différents acteurs ; il est important en effet de chercher à mieux saisir ces critiques, car cette nouvelle demande sociétale est souvent prise comme point de départ pour proposer de nouveaux produits, insuffler de nouvelles voies de recherche et établir diverses réglementations, mais ces mesures sont-elles toujours adaptées ? En premier lieu, on cherche à mieux connaître la perception des risques par les consommateurs en matière de qualité et de sûreté(1) des produits alimentaires et d'environnement. Sont analysés ensuite divers facteurs explicatifs et origines du questionnement envers le type d'agriculture actuel. A partir de là, on examine si les solutions qui sont proposées face aux risques dénoncés et à la crise de confiance paraissent adaptées. Ce travail s'appuie sur une analyse de l'évolution technique de l'agriculture et de l'alimentation, un suivi et une observation des mouvements de critiques du productivisme et des réponses proposées. Une approche globale de ces questions a été choisie, et non celle d'un cas particulier : des études de cas précis existant déjà, il s'avère utile de relier les phénomènes étudiés par des approches de synthèse pouvant permettre une vue d'ensemble plus globale.
Cette analyse de la crise de confiance et du questionnement des consommateurs ne signifie pas que ceux-ci soient non fondés (il suffit de penser aux risques de I'ESB, des allergies ou de l'obésité par exemple), mais que ses modes d'élaboration et ses formes d'expression nécessitent d'être mieux connus, d'autant plus qu'ils jouent un grand rôle dans les réponses recherchées par les divers acteurs.

\section{1 / Quelques estimations des risques perçus par les consommateurs}

Les mutations de l'agriculture dans les décennies d'après guerre ont été dictées par trois objectifs, productivité, efficience et compétitivité. Beaucoup de résultats ont été obtenus comme la diminution du coût relatif de l'alimentation dans le budget des ménages (tableau 2), l'amélioration du niveau de vie général, l'allongement de l'espérance de vie (tableau 3), etc. Mais depuis quelque temps on note une montée de la suspicion, en tout cas une certaine perte de confiance envers la qualité des produits et beaucoup de récriminations envers l'agriculture. Le phénomène ne se limite pas à des cas particuliers où des entorses à la sécurité sanitaire ont provoqué des accidents : des critiques parfois vives s'élèvent de plus en plus souvent contre le modèle de production agricole dans son ensemble et expriment un net rejet du productivisme, ce qui induit pour le secteur la quête de nouvelles légitimités (Miclet et al 1998).

Tableau 2. Le coût de l'alimentation et son évolution : quelques repères (d'après INSEE).

Part du budget consacré à l'alimentation par les ménages ( $\%$ des dépenses totales de consommation)

\begin{tabular}{l|c|c} 
- en 1997 & France & UE à 15 \\
\hline Alimentation seule & 13,9 & - \\
\hline Alimentation + boissons & 16,4 & - \\
\hline Alimentation + boissons + tabac & 17,9 & 17,4
\end{tabular}

- évolution depuis 1949 en France (boissons comprises, mais tabac exclu sauf en 1949)

\begin{tabular}{l|c|c|c}
1949 & 1959 & 1979 & 1997 \\
\hline 44,5 & 34,0 & 20,9 & 16,4 \\
\hline
\end{tabular}

En matière de dépenses deconsommation, il ne faut pas oublier que, si la proportion consacrée à l'alimentation a diminué dans le budget des ménages, le montant total des dépenses alimentaires à monnaie constante (hors inflation) a augmenté entre 1950 et 2000, en raison notamment de la sophistication croissante de l'alimentation.

Evolution comparée du prix des produits agricoles et alimentaires à monnaie constante (Indice 100 en 1970)

\begin{tabular}{l|c|c} 
& 1960 & 1997 \\
\hline Prix agricoles à la production & 108 & 49 \\
\hline Prix alimentaires de détail & 100 & 109 \\
\hline
\end{tabular}

(1) Le terme de sécurité alimentaire, très fréquemment utilisé aujourd'hui, renvoie à deux acceptions différentes : en France et dans les pays occidentaux il évoque le plus souvent des aliments sains, la sécurité en termes de santé publique (food safety). Dans les pays en développement il renvoie généralement aux problèmes d'accès à une nourriture suffisante (food security). Pour éviter l'ambiguïté, dans ce texte on réservera le terme de «sécurité alimentaire» à son sens historique (nourriture suffisante) ; pour rendre compte des questions de «food safety» actuellement souvent sur le devant de la scène, on utilisera de préférence le terme de "sécurité sanitaire des aliments» ou de «sûreté alimentaire» ou encore de «sécurité des aliments

INRA Producti ons Ani males, décembre 2000 
Tableau 3. Evolution de l'espérance de vie (années) à la naissance en France depuis un siècle (source INED).

\begin{tabular}{|c|c|c|}
\hline & Hommes & Femmes \\
\hline 1900 & 43,2 & 46,9 \\
1910 & 49,5 & 53,3 \\
1920 & 49,6 & 53,5 \\
1930 & 54,4 & 59,4 \\
1939 & 56,6 & 62,6 \\
1950 & 63,4 & 69,2 \\
1960 & 67,0 & 73,6 \\
1970 & 68,4 & 75,8 \\
1980 & 70,2 & 78,4 \\
1990 & 72,8 & 80,9 \\
1999 & 74,9 & 82,3 \\
\hline
\end{tabular}

Ces critiques envers les conséquences de la modernisation de l'agriculture ne sont pas récentes, mais étaient naguère plus minoritaires. Ainsi le mouvement qui a donné naissance à l'agriculture biologique date de plusieurs décennies (Viel 1979), mais pendant longtemps son écho est resté limité à quelques groupes associatifs restreints. Dans les années 1970, des questionnements plus larges se sont manifestés à diverses reprises suite notamment à la crise de l'énergie ; puis, dans les années 1980, aux problèmes de surproduction, de coût budgétaire de la PAC et de conflits internationaux liés aux exportations subventionnées. Ils se sont étendus ensuite dans le grand public à partir de la fin des années 1980 avec la montée de la sensibilité envers diverses pollutions (nitrates, lisiers), puis, depuis le milieu des années 1990, avec les questions de sûreté alimentaire liés à l'ESB, à la fabrication des aliments du bétail et aux débats sur les OGM.

En premier lieu, ¡l paraît nécessaire d'avoir une meilleure connaissance du degré de confiance ou de suspicion envers les produits agricoles et alimentaires. En ce domaine il est souvent fait recours à des sondages. Toutefois, s'ils fournissent des indications utiles, ils présentent aussi diverses limites ${ }^{(2)}$. En gardant celles-ci à l'esprit, nous allons présenter les résultats de plusieurs d'entre eux en matière de qualité et de sûreté des produits agricoles et alimentaires.

L'environnement est un domaine où l'héritage que le XXème siècle lègue apparaît parmi les plus négatifs : c'est ce qu'estiment les deux tiers des Européens et même les trois quarts des Italiens et des Allemands de l'Ouest, mais un tiers seulement des Finlandais (tableau 4 ; CE 1999). Toutefois les préoccupations environnementales portent en premier lieu sur la qualité de l'air, non sur l'agriculture. Parmi les grands domaines de préoccupation des Français, une enquête effectuée fin octobre 1999 fait ressortir très majoritairement les problèmes socio-économiques (chômage, exclusion, violence des banlieues) et de façon nettement moindre les problèmes environnementaux ou de qualité des produits alimentaires (tableau 5).

Pour l'agriculture, divers sondages des dernières années sur des questions de qualité, sûreté sanitaire et environnement montrent que l'opinion publique est assez nuancée, bien davantage que ne le donneraient à penser divers dossiers parus dans des médias grand public comme on l'a déjà souligné (Bonny 2000a, tableau 5). Les différences que l'on note entre des sondages effectués à des dates assez proches peuvent s'expliquer par la formulation des questions et par le contexte,

Tableau 4. Classement des domaines où l'héritage légué par le XX'me siècle est jugé «plutôt positif» ou «plutôt négatif» par les habitants de l'UE.

Enquête Eurobaromètre 50, réalisée entre fin octobre et début décembre 1998 auprès de 16224 personnes de l'UE.

Domaines jugés majoritairement positifs ( $\%$ des réponses)

\begin{tabular}{|l|c|c|}
\hline & «plutôt positif» & «plutôt négatif» \\
\hline Liberté & 72 & 21 \\
Qualité de la vie & 58 & 33 \\
Egalité & 54 & 36 \\
Solidarité & 47 & 42 \\
Protection sociale & 47 & 43 \\
Pouvoir d'achat & 46 & 41 \\
\hline
\end{tabular}

Domaines jugés majoritairement négatifs ( $\%$ des réponses)

\begin{tabular}{|l|c|c|}
\hline & «plutôt positif» & «plutôt négatif» \\
\hline Conditions de travail & 42 & 49 \\
Sécurité individuelle & 38 & 52 \\
Moralité, éthique & 33 & 54 \\
Environnement & 27 & 66 \\
Emploi & 24 & 68 \\
\hline
\end{tabular}

Tableau 5. Classement des sujets de préoccupation des Français fin octobre 1999 (IPSN 2000).

«En France, parmi les problèmes actuels suivants, lequel est pour vous le plus préoccupant?"

Somme des thèmes cités en $1^{\text {ère }}$ et en $2^{\text {ème }}$ position (la somme des pourcentages est donc de $200 \%$ )

\begin{tabular}{|l|c|}
\hline & \%deréponses \\
\hline Chômage & 53,8 \\
Misère et exclusion & 34,6 \\
Violence dans les banlieues & 33,4 \\
Dégradation de l'environnement & 19,7 \\
SIDA & 18,8 \\
Déficit de sécurité sociale & 11,5 \\
Qualité des produits alimentaires & 8,8 \\
Sûreté des centrales nucléaires & 8,2 \\
Violence au sein des familles & 7,9 \\
Nuisances sonores & 2,1 \\
Ne sait pas & 1,2 \\
Total 1' et $^{\text {ème }}$ sujet & 200 \\
\hline
\end{tabular}

(2) Ces limites sont en particulier le risque d'artefact quand l'enquêté doit choisir, en un temps très court et hors contexte, une réponse parmi une série d'items proposés sur un sujet complexe; I'influence de la thématique générale et de l'enchaînement des questions sur les réponses obtenues à chacune, et, enfin, le risque de superficialité de l'approche par comparaison à des entretiens approfondis. En revanche, par rapport à ces derniers, les sondages ont l'intérêt de fournir des indications sur de vastes échantillons représentatifs de la population étudiée. Par ailleurs il ne faut pas oublier qu'une opinion déclarée peut différer de l'opinion effective (a fortiori si l'interviewe escompte des
retombées aux résultats de l'enquête et biaise ses réponses pour cela), et peut différer surtout du comportement en situation réelle. INRA Producti ons Ani males, décembre 2000

\author{
Fin 1999, parmi \\ les problèmes \\ actuels les plus \\ préoccupants, \\ la qualité des \\ produits \\ alimentaires était \\ citée en premier \\ par seulement $2,4 \%$ \\ des Français et en \\ second par $6,4 \%$.
}


mais aussi par le caractère très polysémique du terme «qualité»: selon les cas, l'enquêté peut se référer par exemple plutôt à la sûreté sanitaire, ou aux caractéristiques organoleptiques, ou à certaines caracteristiques nutritionnelles. En tout cas, il ressort de ces diverses enquêtes que, dans la deuxième moitié des années 90 , les opinions envers la qualité et la sûreté alimentaire étaient en fait assez éloignées d'une vision catastrophique que d'autres dénonçaient.

Mais en 1999 et 2000 , les questions de pollutions agricoles, de qualité et de sûreté des aliments sont devenues encore plus omniprésentes: OGM, vache folle, listéria, salubrité des aliments pour animaux, etc. ont fréquemment fait la une des médias. L'ensemble de la filière, les pouvoirs publics, les parlementaires, les instances communautaires et diverses associations se sont fortement mobilisés sur ces questions. Quant aux consommateurs, peut-on évaluer leur degré de préoccupation en ce domaine à la même période et son évolution ? En 2000 aussi, comme les années précédentes, les résultats des sondages demeurent variables, même si globalement la sensibilisation augmente. Ainsi une enquête d'avril 2000 sur le thème « les Français et la santé » fait apparaître un très fort pourcentage de satisfaction ( $90 \%$ ) envers la qualité de la nourriture et un fort pourcen- tage de satisfaction ( $69 \%$ ) envers la qualité de l'eau, avec toutefois des écarts sensibles selon les régions (tableau 6) ! Dans la liste des divers choix proposés, " améliorer la qualité de la nourriture » est un objectif jugé prioritaire par seulement $6 \%$ des enquêtés (mais par $17 \%$ des professionnels de la santé) ; il est classé en dernier dans la presque totalité des cas, quels que soient la tranche d'âge, la région, le milieu, le niveau de revenu, etc!

A l'opposé, d'autres sondages récents centrés spécifiquement sur la sécurité alimentaire font ressortir une montée des préoccupations, notamment chez les femmes, mais moins prononcée chez les 25-34 ans (tableau 7). Cette préoccupation semble modifier certains comportements lors de l'achat : les consommateurs portent par exemple une plus grande attention à diverses indications figurant sur les produits. Quels éléments inquiètent le plus les enquêtés en matière de sécurité sanitaire des aliments ? Dans la liste mentionnant divers risques en la matière, vient en tête pour les trois ans à venir l'ESB, puis à un degré moindre les OGM, et ensuite la pollution de l'eau par les nitrates, les listérioses, la rupture de la chaîne du froid, les hormones en alimentation animale, la dioxine (tableau 8). Mais comme les années précédentes, l'opinion que la qualité se dégrade n'apparaît pas largement prédominante, du moins jusqu'à

Tableau 6. Sujets de satisfaction ou de préoccupation des Français dans le domaine santé-environnement (en \% des réponses pour chaque domaine).

Source : sondage «les Français et la santé», avril 2000, CSA/Syndicat de la Presse Quotidienne Régionale, avec deux échantillons : l'un de 2802 personnes représentatif de la population française au niveau national et régional, l'autre de 400 professionnels de la santé (médecins, personnel hospitalier).

«Dans votre région, là où vous vivez, diriez-vous que la situation est-elle plutôt satisfaisante ou plutôt pas satisfaisante dans les domaines suivants liés à votre santé ?»

"Qu'est-ce qui doit être prioritairement amélioré à votre avis ?"

\begin{tabular}{|c|c|c|c|c|}
\hline \multirow{2}{*}{ Domaines mentionnés } & \multicolumn{2}{|c|}{ «Situation } & \multicolumn{2}{|c|}{ «A améliorer prioritairement» pour } \\
\hline & plutôt satisfaisante» & plutôt pas satisfaisante» & l'ensemble des français & les professionnels de la santé \\
\hline - qualité de la nourriture & 90 & 9 & 6* & $17^{*}$ \\
\hline - mode de vie & 84 & 15 & - & - \\
\hline - qualité des infrastructures médicales & 84 & 14 & $18^{*}$ & $31^{*}$ \\
\hline - possibilité d'avoir une activité physique à proximité & 83 & 15 & 10 & 16 \\
\hline - facilité des déplacements & 79 & 20 & 25 & 26 \\
\hline - niveau de bruit environnant & 76 & 24 & 19 & 13 \\
\hline - qualité de l'eau & 69 & 30 & $29 *$ & $18^{*}$ \\
\hline - niveau de stress & 68 & 30 & $16^{*}$ & $28^{*}$ \\
\hline - climat & 67 & 32 & - & - \\
\hline - qualité de l'air & 67 & 32 & $32 *$ & $25^{*}$ \\
\hline
\end{tabular}

* Résultats significativement différents entre l'ensemble de la population et les professionnels de la santé.

\begin{tabular}{|c|c|c|c|}
\hline Réponses concernant la qualité de la no & $\begin{array}{c}\text { ture selon les régions } \\
\text { «Situation } \\
\text { plutôt satisfaisante» }\end{array}$ & $\begin{array}{c}\text { (en \% des réponses pour c } \\
\text { «Situation } \\
\text { plutôt pas satisfaisante» }\end{array}$ & $\begin{array}{l}\text { haque région). } \\
\text { «A améliorer } \\
\text { prioritairement» }\end{array}$ \\
\hline $\begin{array}{l}\text { Alsace-Lorraine Champagne } \\
\text { Aquitaine Poitou-Charentes } \\
\text { Basse et Haute Normandie } \\
\text { Bourgogne Centre Auvergne Limousin } \\
\text { Bretagne Pays de Loire } \\
\text { Franche-Comté Rhône-Alpes } \\
\text { Ile de F rance } \\
\text { Languedoc Roussillon } \\
\text { Midi Pyrénées } \\
\text { Nord Picardie } \\
\text { PACA Corse } \\
\text { E nsemble (F rance) }\end{array}$ & $\begin{array}{l}90 \\
95 \\
93 \\
92 \\
90 \\
92 \\
84 \\
97 \\
97 \\
84 \\
92 \\
90\end{array}$ & $\begin{array}{r}9 \\
5 \\
7 \\
8 \\
9 \\
8 \\
14 \\
3 \\
3 \\
15 \\
8 \\
9\end{array}$ & $\begin{array}{r}8 \\
7 \\
4 \\
6 \\
12 \\
4 \\
5 \\
5 \\
4 \\
11 \\
6 \\
6\end{array}$ \\
\hline
\end{tabular}

I NRA Productions Ani males, décembre 2000 
l'automne 2000 où la crise liée à l'ESB fait augmenter les craintes liées à la transmission de cette maladie.

Les enquêtes montrent que si certaines techniques agricoles sont souvent critiquées actuellement, les agriculteurs conservent un bon capital de sympathie. Ils sont assez fréquemment exonérés de la pleine responsabilité de la dégradation de la qualité ou de l'environnement : les contraintes économiques sont souvent jugées comme déterminantes en la matière (Bonny 2000a). Ainsi, dans un sondage récent réalisé par IPSOS à la demande du Ministère de l'Agriculture fin juin 2000 auprès de 2600 personnes, 80 à $85 \%$ des enquêtés estiment que les agriculteurs de leur région remplissent bien les objectifs qui leur sont donnés, c'est-à-dire dans la liste proposée « maintenir la production des produits traditionnels du terroir », «fournir des produits alimentaires de qualité », « maintenir une vie rurale active » (tableau 9). La majorité des enquêtés (respectivement 70 et $62 \%$ ) pensent aussi que l'entretien des paysages et la préservation de l'environnement - autres objectifs listés - sont également bien réalisés par les agriculteurs. A peine un tiers seulement des répondants jugent ces objectifs mal remplis, et les principales causes - parmi celles proposées dans le sondage - en sont pour les enquêtés : le manque de moyens financiers », «le manque de temps ou de main d'œuvre », plus rarement «le fait de ne pas se sentir concernés » ou de « privilégier le rendement ».
Mais en 2000, les risques alimentaires sont souvent jugés en augmentation. Le fait d'être mieux informé intervient pour beaucoup dans cette perception.

Tableau 7. Perception en 2000 de l'évolution des risques en matière de sûreté des aliments.

7.1 / Préoccupations en matière de sécurité sanitaire des aliments

Sondage ISL / Revues LSA et «Objectif Risk Zéro» des 23-24 mars 2000 (1000 personnes).

\begin{tabular}{|l|c|c|}
\hline & OUI & NON \\
\hline «Aujourd'hui êtes-vous plus préoccupés qu'hier par les problèmes de sécurité alimentaire? » & 52,4 & 47,6 \\
«Faites-vous plus attention... » & 89,1 & 10,8 \\
- au type de produits que vous achetez & 87,3 & 12,3 \\
- à l'origine des produits que vous achetez & 85,6 & 13,7 \\
- aux mentions qui figurent sur les emballages & 76,8 & 23,2 \\
- aux labels mentionnés sur les emballages (type label rouge, agriculture biologique) & 69,3 & 30,4 \\
- aux marques que vous achetez &
\end{tabular}

\section{2 / Evolution perçue des risques par rapport à il y a 10 ans selon divers sondages}

a / Sondage BVA-ANIA (association nationale des industries alimentaires), 11-21 avril 2000, 1007 personnes. ( $\%$ des réponses par colonne, le total étant de $100 \%$ pour chaque colonne)

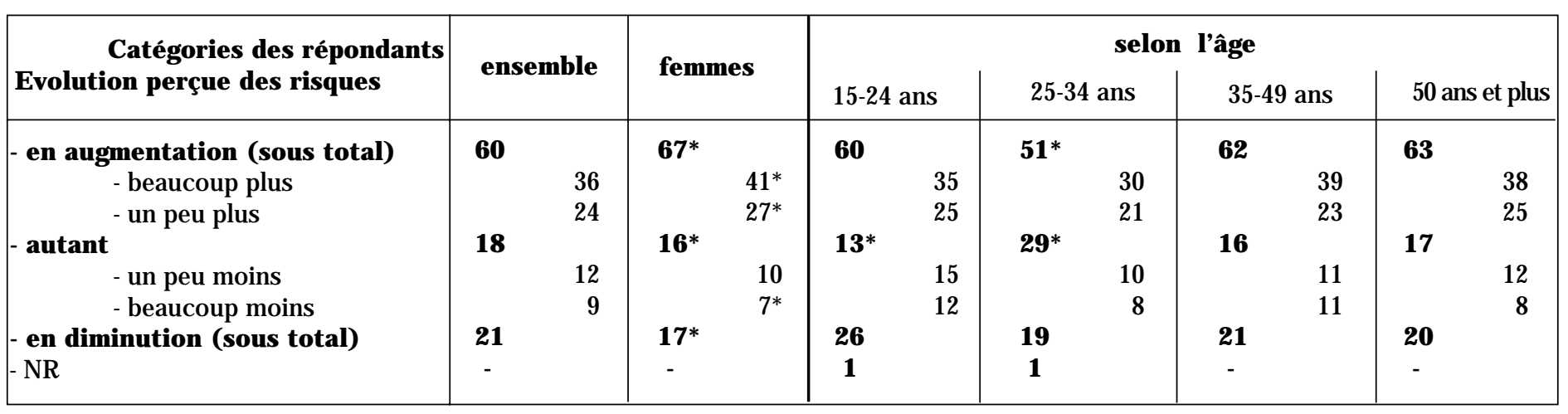

* écarts significatifs par rapport à l'ensemble de l'échantillon

b / Sondage IFOP - Libération du 20 au 21 juillet 2000, échantillon de 952 personnes.

«Par rapport à il y a 10 ans, diriez-vous que la situation en matière de qualité des produits alimentaires en France s'est plutôt améliorée ou s'est plutôt détériorée ?»

\begin{tabular}{|c|c|c|c|c|}
\hline & plutôt améliorée & est restée inchangée & plutôt détériorée & ne se prononce pas \\
\hline en $\%$ des réponses & $\mathbf{4 8}$ & 6 & $\mathbf{4 5}$ & 1 \\
\hline
\end{tabular}

c/ Sondage IPSOS / Etats Généraux de l'Alimentation, 6-7 octobre 2000, échantillon de 1025 personnes.

- «Diriez-vous que depuis 10 ans, les produits alimentaires présentent en général pour la santé des consommateurs ...»

\begin{tabular}{|c|c|c|c|c|}
\hline & ...plus de risques & ...ni plus, ni moins & ...moins de risques & ne se prononce pas \\
\hline en $\%$ des réponses & $\mathbf{3 0}$ & 49 & $\mathbf{2 0}$ \\
\hline
\end{tabular}

- Influence de l'information et des contrôles

\begin{tabular}{|l|l|}
\hline & $\%$ \\
\hline - les risques ne sont pas plus nombreux mais ils sont mieux connus car les contrôles sont plus fréquents \\
- les risques sont plus nombreux qu'avant & 76 \\
- Ne se prononce pas & 23 \\
\hline
\end{tabular}


En définitive, en 1999 et 2000, une partie des consommateurs se disent préoccupés par les risques en matière de sécurité sanitaire des aliments, mais une partie seulement (hors du cas de l'ESB à l'automne 2000). Un sondage d'avril 2000 montre que les risques sont perçus comme provenant surtout du secteur amont (industriels fournissant engrais ou aliments du bétail), dans une moindre mesure des fabricants de produits alimentaires, puis plus faiblement des distributeurs, des agriculteurs et des consommateurs eux-mêmes (tableau 10).

Les jugements varient quelque peu selon les divers groupes sociaux (CREDOC 1998), mais surtout selon les valeurs et la culture. Aussi faut-il bannir l'expression si courante "LE (ou LES) consommateur(s) demande(nt)... », et prendre en compte leurs diverses catégories. Dans ce cadre, il faut souligner les variations notables d'opinions entre pays de I'UE, du fait de sa diversité culturelle, ce qui peut expliquer des difficultés à prendre parfois des positions communes. On peut l'illustrer par les résultats d'un sondage Eurobaromètre effectué à la demande de la DG XXIV (Politique des Consommateurs) de la Commission Européenne en mai-juin 1998 montrant les différences de vue sur la question «où trouve-t-on les produits alimentaires les plus sûrs ?» (CE 1998). Dans certains pays (Finlande, Allemagne, France, Autriche, Luxembourg, Suède, Portugal) on fait confiance en premier lieu aux «fermiers et petits producteurs»; dans d'autres au contraire (Pays-Bas, Royaume-Uni, Espagne, Irlande,
Danemark) les supermarchés sont placés en tête, avec une importance variable des autres lieux d'achat comme les marchés (figure 1). Le classement des lieux "où l'on trouve les produits les plus sûrs» est par contre moins influencé par l'âge, le sexe, la catégorie socioprofessionnelle. En matière de biotechnologies, le profil des positions - c'est-à-dire la part relative des supporters, des partisans nuancés (percevant certains risques) et des opposants - diffère aussi entre les pays européens, ainsi qu'entre les USA et l'Europe (CE 1997 et 2000, Zechendorf 1998, Gaskell et al 1999 et 2000), même si un certain rapprochement s'est esquissé fin 1999 et en 2000 (Hornig Priest 2000). Cette diversité s'explique notamment par le déterminant culturel qui influe sur le degré de confiance dans les autorités administratives, sur l'attitude envers l'artificialisation de la nature et sur la foi dans le développement économique.

Ainsi, on relève effectivement des craintes en matière de sécurité sanitaire des aliments ou un mécontentement en matière de leur qualité. Mais, malgré l'omniprésence des dossiers parfois fort alarmants sur ces thèmes dans de nombreux médias, cela demeure relativement nuancé et ne concerne pas la majorité de la population, au moins jusqu'à la crise de novembre 2000 . Les critiques exprimées semblent se focaliser plus fréquemment sur les techniques utilisées en agriculture et en agro-alimentaire que sur la responsabilité des agriculteurs eux-mêmes.

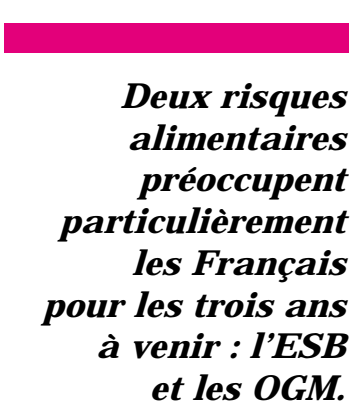

Tableau 8. Les trois risques jugés les plus importants pour les trois années à venir en matière de sûreté alimentaire (même sondage BVA-ANIA que tableau 7.2.a, en avril 2000).

\begin{tabular}{|l|c|c|c|c|}
\hline \multicolumn{1}{|c|}{ \% de personnes le citant } & en 1 & en $\mathbf{2}^{\text {ème }}$ & en $\mathbf{3}^{\text {'me }}$ & total \\
\hline Liste de risques cités dans l'enquête & & & & \\
ESB ou maladie de la vache folle & 27 & 16 & 10 & $\mathbf{5 2}$ \\
OGM & 14 & 10 & 9 & $\mathbf{3 2}$ \\
Pollution de l'eau du robinet par les nitrates & 9 & 8 & 10 & $\mathbf{2 7}$ \\
Listéria ou listériose & 9 & 11 & 7 & $\mathbf{2 6}$ \\
Rupture de la chaîne de froid entre le fabricant et le point de vente & 7 & 7 & 10 & $\mathbf{2 4}$ \\
Hormones données aux animaux & 5 & 9 & 7 & $\mathbf{2 2}$ \\
Dioxine & 8 & 7 & 7 & $\mathbf{2 1}$ \\
Antibiotiques donnés aux animaux & 4 & 6 & 6 & $\mathbf{1 7}$ \\
Epandage des boues d'épuration sur les champs cultivés & 4 & 6 & 6 & $\mathbf{1 6}$ \\
Traitements chimiques sur les cultures & 3 & 5 & 7 & $\mathbf{1 4}$ \\
Salmonelles ou salmonellose & 2 & 5 & 6 & $\mathbf{1 3}$ \\
Mauvaise conditions de conservation des aliments chez les consommateurs & 2 & 4 & 4 & $\mathbf{1 0}$ \\
Dépassement des dates limites de vente & 2 & 2 & 4 & $\mathbf{9}$ \\
Colorants ou conservateurs contenus dans les aliments & 2 & 2 & 2 & $\mathbf{6}$ \\
\hline
\end{tabular}

Tableau 9. Les agriculteurs remplissent-ils les missions qu'on leur attribue ? Une évaluation par l'ensemble de la population (réponse en \% pour chaque mission, total $100 \%$ par ligne). ST : sous-total

Sondage IPSOS/Ministère de l'agriculture :"Les Français et l'agriculture ”, réalisé du 21 au 30 juin 2000 sur un échantillon de 2600 personnes interrogées par téléphone, suivant la méthode des quotas.

\begin{tabular}{|l|c|c|c|c|c|c|c|}
\hline Missions attribuées mentionnées dans le sondage & très bien & assez bien & ST bien & assez mal & très mal & ST mal & sans opinion \\
Maintenir la production des produits traditionnels du terroir & 24,3 & 60,7 & $\mathbf{8 5 , 0}$ & 10,2 & 1,7 & $\mathbf{1 1 , 9}$ & 3,1 \\
Fournir des produits alimentaires de qualité & 21,9 & 62,9 & $\mathbf{8 4 , 8}$ & 9,9 & 2,5 & $\mathbf{1 2 , 4}$ & 2,9 \\
Maintenir une vie rurale active & 18,5 & 61,0 & $\mathbf{7 9 , 5}$ & 13,4 & 2,4 & $\mathbf{1 5 , 8}$ & $\mathbf{4 , 7}$ \\
Entretenir le paysage et aménager les campagnes & 15,4 & 54,2 & $\mathbf{6 9 , 6}$ & 19,9 & 8,1 & $\mathbf{2 8 , 0}$ & 2,5 \\
Préserver l'environnement (eau, air, sols, espèces végétales et animales) & 10,6 & 51,9 & $\mathbf{6 2 , 5}$ & $\mathbf{2 4 , 9}$ & 8,6 & $\mathbf{3 3 , 5}$ & 4,0 \\
\hline
\end{tabular}




\section{2 / Facteurs explicatifs et origines des interrogations envers le type d'agriculture actuel}

II ne suffit pas que des dangers ou des problèmes existent dans un secteur, une activité ou un produit pour qu'une mobilisation se déclenche et entraîne la mise sur agenda de cette question et la recherche active de solutions ! II est nécessaire que le risque soit perçu, pris en compte et mobilise des acteurs, des associations ou des organismes susceptibles de se faire entendre, et par là suscite l'intérêt de façon assez large afin de mettre en mouvement des moyens de résolution (Gilbert 1998, Chateauraynaud et Torny
1999) : ce processus de mise sur agenda est assez complexe et subtil. II peut aboutir à focaliser l'attention sur des risques faibles en termes de morbidité et de mortalité alors que d'autres à l'impact bien plus important suscitent moins de mobilisation. A l'opposé, même quand l'émergence d'un risque a été bien signalée, l'alarme peut ne pas être entendue et le problème peut être jugé marginal (cf. par exemple diverses dénégations envers la gravité du SIDA dans la première moitié des années 1980, ou récemment face au changement climatique anthropique). De ce fait il est nécessaire de prendre en considération non seulement les faits «objectifs» en eux-mêmes, mais aussi divers facteurs sociétaux ou sociopolitiques expliquant la sensibilisation à l'égard de certains risques.

Figure 1. Lieux où l'on trouve les produits alimentaires les plus sûrs selon les habitants des divers pays de l'UE (enquête Eurobaromètre 1998, cf. CE 1998)

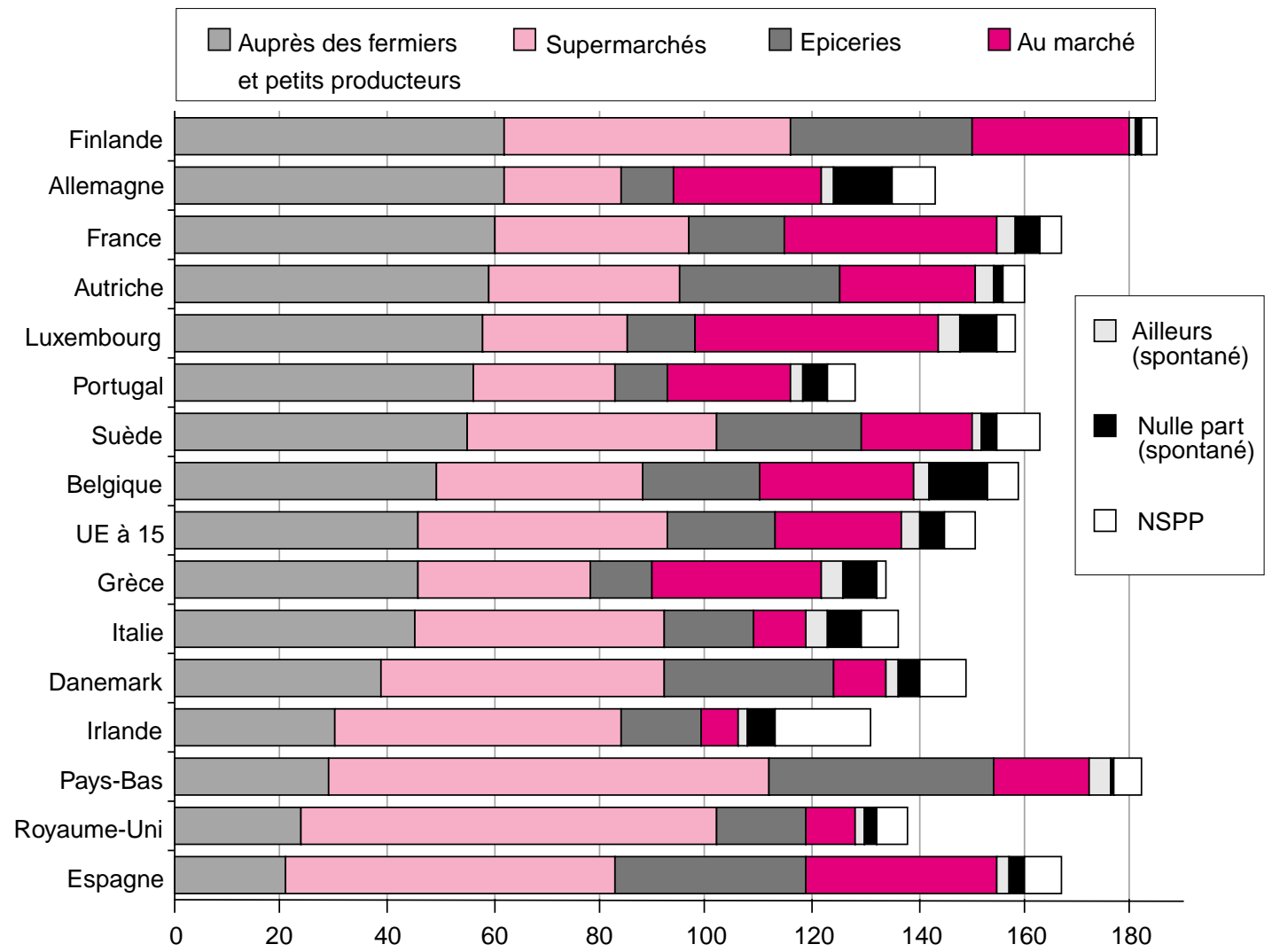

Tableau 10. De quels acteurs de la filière proviennent surtout les risques en matière de sûreté alimentaire selon les consommateurs ? (même sondage BVA-ANIA qu'au tableau 7.2.a, avril 2000).

Classement depuis la note 10 (= peut tout à fait être à l'origine d'un risque)

jusqu'à la note 0 (= ne peut en aucun cas être à l'origine d'un risque).

\begin{tabular}{|l|c|c|c|c|c|c|}
\hline Catégories des répondants & ensemble & femmes & $15-24$ ans & $25-34$ ans & $35-49$ ans & 50 ans et plus \\
\hline Note de responsabilité attribuée à & & & & & & \\
- industriels fabricant engrais ou aliments du bétail & $\mathbf{8 , 1}$ & 8,1 & $7,5^{*}$ & 8,2 & $8,4^{*}$ & 8,0 \\
- fabricants de produits alimentaires & $\mathbf{6 , 6}$ & 6,8 & $6,2^{*}$ & 6,7 & 6,9 & 6,5 \\
- transporteurs de produits alimentaires & $\mathbf{5 , 5}$ & $5,8^{*}$ & $7,7^{*}$ & 5,6 & 5,5 & 5,7 \\
- distributeurs / magasins & $\mathbf{5 , 3}$ & 5,4 & $4,4^{*}$ & 5,6 & 5,3 & 5,5 \\
- agriculteurs ou éleveurs & $\mathbf{5 , 2}$ & 5,1 & $4,6 *$ & 5,4 & 5,4 & 5,1 \\
- consommateurs & $\mathbf{4 , 8}$ & 4,9 & $3,9 *$ & 4,6 & 4,9 & $5,1^{*}$ \\
\hline Taille de l'échantillon & 1007 & 523 & 168 & 184 & 275 & 380 \\
\hline
\end{tabular}

* écart significatif par rapport à la moyenne de l'échantillon 


\section{1 / Aspects économiques et structurels de remise en cause du modèle productiviste}

La montée des débats sur le modèle productiviste est liée à diverses limites apparues du fait même de la poursuite du modele productif agricole des décennies d'après-guerre sur sa lancée, sans un infléchissement adaptatif suffisamment précoce en divers domaines. Ces limites sont souvent évoquées : excédents agricoles, coûts budgétaires des soutiens, impacts sur l'environnement, inégalités spatiales de répartition des exploitations avec de fortes concentrations en certaines zones entraînant des pollutions et à l'opposé déprise agricole en d'autres régions. De leur côté l'urbanisation, les transformations de la structure de la population et l'évolution socio-économique et sociétale d'ensemble ont induit des modifications de la demande adressée à l'agriculture pour les produits alimentaires, mais aussi pour les fonctions remplies, les méthodes utilisées, les paysages créés, etc. Dans le même temps la concurrence entre les diverses entreprises du secteur et entre pays agro-exportateurs s'est fortement avivée.

Les maîtres mots dans la filière agro-alimentaire durant les dernières décennies ont été et sont encore la recherche d'une réduction des coûts de production du fait de cette concurrence. Cet impératif est particulièrement fort dans le secteur agricole et dans les entreprises où sont fabriqués les matières premières et les produits intermédiaires utilisés par des secteurs aval. En effet, si une différenciation de qualité peut permettre de vendre à prix plus élevé au consommateur final, cela n'est pas toujours le cas plus en amont. En outre, le secteur de la distribution alimentaire, très puissant et concentré, impose de bas prix à ses fournisseurs pour leur référencement et l'accès à ses linéaires. D'où, en amont, la recherche poussée à l'extrême de matières premières les moins onéreuses possibles et d'une baisse des coûts. Or cela peut parfois engendrer certains problèmes de sûreté alimentaire comme le montrent les exemples de I'ESB (on a réduit la température et la pression de chauffage des produits d'abattoir recyclés en farines animales), de I'emploi d'antibiotiques ou d'hormones, etc. Certes des problèmes de frelatage de denrées alimentaires existent depuis des siècles et même des millénaires. Mais le contexte actuel de surproduction agricole en $\mathrm{E}$ urope occidentale, les a rendus aujourd'hui totalement inacceptables par le grand public et accroît son indignation à leur égard : la présidente de I'UFC-Que Choisir exprime ainsi qu'il est inconcevable aujourd'hui de «prendre des risques en mangeant».

Cette recherche poussée à l'extrême d'une réduction des coûts directs peut induire aussi des formes de production sanitairement risquées ou éthiquement critiquables comme l'emploi d'antibiotiques pour favoriser la croissance, ou la très forte densité d'animaux en élevage avicole. Cela paraît aisément choquant vu de l'extérieur - c'est-à-dire sans considérer les contraintes de prix et de concurrence : l'économie réalisée, si l'on inclut les coûts indirects et les externalités, peut sembler ne pas toujours justifier les formes d'élevage pratiquées dénoncées comme trop productivistes.

Certes les risques sanitaires alimentaires ont fortement diminué en bien des domaines grâce à une nette amélioration de l'hygiène et grâce à l'éradication de diverses maladies endémiques des animaux. Mais d'autres facteurs sont susceptibles de les accroître, notamment pour les produits les plus en aval :

- la recherche de procédés et de matières premières à bas coût pour faire face à la concurrence ;

- I'allongement et la complexification de la chaîne de transformation en lien avec le très grand nombre d'ingrédients utilisés et la croissance des plats tout préparés ;

- le développement de l'emploi de la surgélation et réfrigération induisant la multiplication des points critiques où la chaîne du froid peut être rompue :

- l'augmentation enfin de la cuisine collective qui amplifie considérablement l'impact d'un éventuel accident, etc.

Tableau 11. Quels problèmes mondiaux actuels paraissent les plus inquiétants ? (Sondage Enjeux-les Echos / SOFRES, des 24-27 mai 2000; échantillon de 1000 personnes représentatif de la population française de plus de 18 ans, interrogé en face à face par un enquêteur, méthode des quotas).

(en \% des réponses, le total étant supérieur à 100 car les personnes interrogées ont pu donner trois réponses). \begin{tabular}{|l|l|}
\hline Liste des problèmes proposées classés selon les résultats & $\%$
\end{tabular} \begin{tabular}{|l|l|l}
\hline La dégradation de l'environnement & 51
\end{tabular}

La faim dans le monde

Le développement du SIDA

La sécurité alimentaire

Le développement des réseaux criminels internationaux, comme la mafia russe

Les mouvements terroristes

Les risques de guerre

La spéculation financière

La mondialisation de l'économie

Le pouvoir croissant des grandes entreprises face aux Etats

La menace des virus informatiques

La nécessité pour les pays occidentaux d'importer de la main d'œuvreétrangère au cours des 10 prochaines années Le rôle central des nouvelles technologies et d'Internet dans l'économie

Sans réponse 
De la sorte diverses alarmes sanitaires fortement médiatisées ont terni l'image du secteur et réduit la confiance, du moins dans une partie de la population, et l'image de la production agricole et alimentaire est devenue moins bonne. Dans une enquête de mai 2000 , parmi les divers grands problèmes perçus comme les plus inquiétants dans le monde, la sûreté alimentaire fait partie des quatre premiers mentionnés par les Français (tableau 11), à une proportion relativement proche de la faim dans le monde ! L'inquiétude en la matière apparaît plus forte chez les 35-49 ans, les commerçants/artisans/industriels et les ouvriers, et pour ceux ayant un niveau d'études inférieur au BEPC. A contrario c'est un domaine où près des trois quarts des enquêtés pensent qu'il n'y a pas suffisamment de règles, notamment les jeunes, les chômeurs et les employés, les personnes proches des écologistes et celles proches du parti communiste (tableau 12).

Aussi de nouveaux objectifs de qualité, sécurité sanitaire, préservation de l'environnement sont-ils aujourd'hui constamment mis au premier plan afin de restaurer l'image du secteur et la confiance.

\section{2 / Origines et facteurs de la crise de confiance envers l'agriculture et l'alimentation modernes}

\section{a / Une industrialisation de l'agriculture et de l'alimentation assez méconnue}

L'anxiété envers l'alimentation, qui est le terreau des peurs alimentaires, provient pour certains sociologues de la distance croissante entre producteurs et consommateurs. C. Fischler (1993) note que le produit alimentaire de grande consommation est un objet de plus en plus manufacturé qui ne présente pas le caractère familier qu'il possède dans une situation de production et de consommation locale : il vient d'ailleurs, a subi des transformations dont on ne connaît pas la nature, a été manipulé par des mains inconnues et contient peut-être des substances mystérieuses. Or l'aliment est un produit particulier : comme on l'ingère, on le fait en quelque sorte devenir une part de soi-même, d'où cette croyance selon laquelle «on est ce qu'on mange». Comme par ailleurs beaucoup pensent aujourd'hui «on ne sait plus ce qu'on mange», cela génère un trouble. A cela on peut ajouter la mystification publicitaire, même si le fait d'en être averti devrait la déjouer : la publicité pour les produits alimentaires donne généralement de l'agriculture une image fort bucolique bien différente de la réalité de la production actuelle. D'où le gap quand les consommateurs découvrent les coulisses, par exemple le recyclage de cadavres d'animaux malades en farines animales consommées par des vaches!

Enfin il convient de prendre en considération que la qualité de l'alimentation perçue renvoie à un ensemble complexe de qualités attendues, composé de six aspects : nutritionnels, sanitaires, fonctionnels, organoleptiques, sociaux et symboliques (Cazes-Valette 1998) ; or ces derniers ne sont pas les moindres.

\section{b / La perception des risques}

Les risques en matière de sûreté alimentaire font l'objet d'une perception très différente selon les acteurs : les experts du secteur jugent en général faibles ceux liés aux methodes de production actuelles, hormis le cas de I'ESB et de l'emploi d'antibiotiques comme additifs en alimentation des volailles. De son côté une partie du grand public fait preuve d'inquiétudes fortes et certaines associations ou groupes les dénoncent avec véhémence. Comment expliquer ces différences de perception? On ne peut se contenter de les attribuer au seul écart d'implication économique ou de responsabilité : chercheurs, vulgarisateurs et membres des Comités d'expertise défendraient le fruit de leur travail (e.g. les résultats obtenus en termes d'efficacité) tandis que médias, essayistes et grand public ignorant les contraintes technico-économiques et la vive compétition dans la filière, se scandaliseraient de leurs conséquences. On ne peut non plus l'imputer simplement, comme on l'entend souvent, au manque de formation en la matière, voire à l'irrationalité du grand public. Diverses recherches ont en effet permis de mieux appréhender la perception des risques. L'expert les évalue en fonction deux composantes : la probabilité qu'un événement indésirable survienne et la gravité de ses conséquences - en matière de sécurité sanitaire on se référera par exemple à la mortalité annuelle. Le public prend lui en compte tout un ensemble d'autres facteurs pour juger les risques comme l'ont montré de nombreux travaux (Slovic 1987, Morgan 1993, Slovic et al 1995, Powell 1998) :

Tableau 12. Demande de réglementation selon les secteurs (même sondage que tableau 11). "Dans chacun des domaines suivants, pensez-vous qu'il y a suffisamment ou pas suffisamment de règles pour encadrer ce que font les entreprises ?»

(en \% des réponses pour chaque domaine, donc par ligne)

\begin{tabular}{|l|c|c|c|}
\hline Domaines classés selon les résultats & Suffisamment & Pas suffisamment & Sans opinion \\
- respect de l'environnement & 13 & 79 & 8 \\
- sécurité alimentaire & 22 & 71 & 7 \\
- Internet & 17 & 64 & 19 \\
- droits des salariés & 29 & 61 & 10 \\
- marchés financiers & 21 & 55 & 24 \\
- commerce international & 25 & 51 & 24 \\
\hline
\end{tabular}

\section{La perception du risque par le public englobe bien d'a utres critères que la seule probabilité de survenue d'un événement indésirable et la gravité de ses conséquences.}


- la connaissance que la personne en a, et le caractère plus ou moins familier du risque; ce qui est invisible et non contrôlable (radioactivité, OGM) est particulièrement anxiogène ;

- l'incertitude scientifique. En cas de controverse on soupconne particulièrement que ceux qui les minimisent aient des intérêts dans le domaine, comme pour l'amiante, ou soient tenus de le faire pour éviter une crise, comme pour l'ESB en Grande-B retagne à la fin des années 1980 (Loder 1999), d'où la crise de confiance «on nous cache quelque chose»;

- la possibilité de contrôle du risque par ceux qui y sont exposés, le sentiment de maîtrise étant essentiel ;

- le caractère volontaire ou non de l'exposition: un risque que l'on ne peut que très difficilement éviter suscite plus d'indignation qu'un autre plus aisé à contourner ou que l'on choisit soi-même :

- l'existence d'avantages - ou non - pour la personne exposée : un risque qui procure un avantage à celui qui le fait courir mais aucun à celui qui le court a un facteur d'indignation élevé :

- le délai d'apparition des conséquences indésirables : on acceptera souvent plus facilement de prendre un risque important (tabagisme, bronzage excessif) si ses effets paraissent fort lointains :

- l'allure de catastrophe : un accident touchant diverses personnes au même lieu au même moment a plus d'impact que des accidents individuels disséminés, même si la somme des personnes touchées est bien moindre :

- la proximité : un risque proche frappe davantage que des catastrophes lointaines bien plus graves.

De la sorte, des pratiques individuelles dont le bilan est lourd (tabagisme, alimentation déséquilibrée, conduite automobile) suscitent moins d'inquiétude que les nitrates ou le génie génétique qui sont moins connus, non observables, difficiles à contrôler et pour lesquels l'exposition ne résulte pas d'un choix individuel. L'acceptabilité dépend ainsi de nombreux facteurs liés à la perception des risques, mais aussi fortement de l'importance des bénéfices qui les justifient ou les compensent.

Le rôle des médias est notable pour faire connaître certains risques que des opérateurs souhaiteraient taire ou minimiser pour éviter une chute de leur réputation. Mais dans les médias règne aussi une très vive concurrence, et la quête d'audience et de la primeur d'annonce peut conduire à faire de la surenchère et à dramatiser les faits, notamment dans des titreschocs exagérés traduisant mal la complexité d'une question. Le milieu journalistique paraît ainsi enclin à dénoncer avec véhémence " ce qu'on vous cache » ou à « révéler en avant-première les bilans alarmants qu'il a pu se procurer $\gg . . . E n$ revanche, quand tous les risques auront été écartés dans un produit ou un processus, l'écho donné sera bien faible en général, parfois même absent, d'où la persistance de la suspicion. Par exemple l'huile de colza, accusée en 1974 de contenir de l'acide érucique (suspecté d'induire des risques cardiaques), n'a jamais pu redorer son blason même après élimination de cet acide gras et malgré ses autres qualités nutritionnelles ! En retour, la crainte d'alarmes disproportionnées et de panique peut conduire des responsables à minorer et sous-estimer cerINRA Productions Ani males, décembre 2000 tains problèmes, comme cela fut le cas avec I'ESB au Royaume-Uni avant le 20 mars 1996 (The BSE Inquiry 2000). Cet exemple montre combien le suivi d'une déontologie bien plus rigoureuse de part et d'autre serait nécessaire pour éviter des enchaînements fort dommageables ; mais les enseignements tirés seront-ils réellement mis en pratique?

\section{3 / Des doutes envers les institutions, le progrès et l'évolution socio-économique}

L'acceptabilité des risques dépend aussi fortement de la confiance dans les institutions. "The perceived risk of biotechnology will be significantly influenced by trust in the system that produces it. (...). Components of the relationship that builds trusts, or distrust, is the extent to which an individual feels affiliated with the system, agrees with the distribution of decisionmaking power, and shares the values enabled by the system » (E spey 1998). Cette question est particulièrement aiguë depuis quelques années en Europe occidentale, les affaires du sang contaminé et de I'ESB ayant conduit à penser que pour préserver des intérêts économiques on faisait courir des risques. Le baromètre de I'IPSN fait ainsi apparaître une confiance plutôt faible dans les autorités et une certaine suspicion envers ce qui est dit sur les dangers : en octobre 1999, en matière d'actions de protection des personnes pour les produits alimentaires, les nitrates/pesticides et les OGM, 35 à $45 \%$ des enquêtés n'avaient pas confiance dans les autorités françaises, 31 à $36 \%$ avaient plus ou moins confiance et seulement 19 à $29 \%$ avaient confiance (tableau 13). En outre diverses innovations récentes sont perçues comme sans utilité pour la société en général, sauf pour les firmes qui les promeuvent, d'où un rejet si des risques éventuels sont suspectés comme pour les OGM (Boy 1999, Valceschini 1999, Gaskell et al 2000).

Les critiques envers le modèle productiviste, ou la suspicion envers la qualité et la sécurité des produits, sont exprimées fréquemment par des arguments d'ordre sanitaire ou environnemental, mais cela peut renvoyer aussi à d'autres dimensions. Le rejet des OGM paraît ainsi souvent témoigner de la cristallisation d'inquiétudes envers l'évolution socioéconomique générale (concentration et pouvoir croissants des firmes, mondialisation, etc.), même si l'on met plus fréquemment en avant des risques sanitaires ou environnementaux qui ont plus de chance de susciter une adhésion générale et qui par ailleurs sont souvent les seuls admis dans les négociations internationales (Bonny 2000b)

On observe aussi parfois plus largement une certaine perte de confiance envers le progrès. Certaines innovations ou certaines recherches scientifiques paraissent parfois dénuées de «sens» (Paillotin et al 1999), ce qui contribue à une attitude de réserve à leur égard. Mais, de fait, l'histoire montre qu'il y a toujours eu une relation complexe des hommes avec les techniques nouvelles, I'innovation et les sciences : admiration et rejet cohabitent souvent. 


\section{3 / Les réponses sont-elles adaptees?}

\section{1 / Les solutions mises en œuvre face aux risques dénoncés et à la crise de confiance}

Face aux critiques, une première attitude chez certains acteurs du secteur $a$, naguère, souvent été de les ignorer ou de les condamner comme irrationnelles et/ou fruits de l'ignorance. II est alors proposé en général de mieux informer et «éduquer» le grand public ; a contrario, on insiste aussi souvent sur les risques importants qu'on prend soi-même délibérément ou par inattention (fumer beaucoup, laisser se réchauffer des surgelés avant de les stocker, omettre de se laver les mains avant les repas, etc.).

Une deuxième réponse est de prendre en considération la nouvelle demande qui s'exprime. Cette attitude est désormais la plus courante car, en économie de marché, il paraît difficile de refuser d'entendre la demande ou le client, a fortiori en situation de vive concurrence et de large gamme de choix ! Ainsi par exemple en 1999, devant la montée de l'opposition aux OGM, la plupart des grandes entreprises de la transformation ou de la distribution ont annoncé les unes après les autres qu'elles n'en utilisaient plus desormais (Bayle et al 1999). De son côté, le souci de sécurité sanitaire s'impose compte tenu du risque commercial de dégradation de l'image de marque et du risque penal en cas de problème.
De la sorte, aux objectifs de baisse des coûts et d'amélioration de la productivité de naguère, se sont ajoutés désormais de nouveaux impératifs pour la production et la transformation:

- la qualité, en particulier en termes de composition, d'aptitude à la transformation, de texture, de saveur, d'arômes, de valeur-santé, etc ;

- la sûreté sanitaire d'autant plus que les méthodes de détection s'affinent toujours davantage ;

- la préservation de l'environnement et la réduction des pollutions :

- la recherche d'une meilleure acceptabilité sociale des techniques utilisées (e.g. bien-être animal).

Ces nouveaux impératifs ressortent par exemple dans les axes de recherche développés par les organismes scientifiques (Feillet 1998, INRA 1999) ou que cherchent à inciter les appels d'offre de la Commission Européenne. Ils se traduisent aussi par l'établissement de nombreuses réglementations et de normes en matière sanitaire et environnementale et par le développement de la traçabilité et de l'étiquetage. Par ailleurs les entreprises de l'agro-alimentaire affinent la différenciation des produits et la segmentation des marchés afin de mieux adapter leur offre aux diverses catégories de clients et à leurs desiderata spécifiques.

\section{2 / Des réponses adaptées ?}

\section{a / Les difficultés et les écueils pour répondre à la demande}

Les professionnels de la filière sont dé-sormais extrêmement attentifs à «traquer»les

Tableau 13. Confiance dans les autorités françaises pour les actions de protection des personnes dans divers domaines et sentiment qu'on dit la vérité sur les dangers qu'ils représentent (baromètre IPSN, sondage BVA effectué les 25-30 octobre 1999 auprès d'un échantillon représentatif de 1015 personnes ; IPSN 2000).

\begin{tabular}{|l|c|c|c|c|c|c|}
\hline \multirow{2}{*}{ \% des répondants } & \multicolumn{3}{|c|}{ font confiance } & \multicolumn{3}{c|}{ estiment qu'on dit la vérité } \\
\cline { 2 - 6 } & $\begin{array}{c}\text { NON } \\
\text { (pas du tout } \\
\text { ou pas vraiment) }\end{array}$ & plus ou moins & $\begin{array}{c}\text { OUI } \\
\text { (tout à fait } \\
\text { ou assez) }\end{array}$ & $\begin{array}{c}\text { NON } \\
\text { (pas du tout } \\
\text { ou pas vraiment) }\end{array}$ & plus ou moins & $\begin{array}{c}\text { OUI } \\
\text { (tout à fait } \\
\text { ou assez) }\end{array}$ \\
\hline Nitrates et pesticides & $\mathbf{4 4 , 2}$ & $\mathbf{3 4 , 1}$ & $\mathbf{1 8 , 6}$ & $\mathbf{5 1 , 6}$ & $\mathbf{2 9 , 0}$ & $\mathbf{1 6 , 0}$ \\
Manipulations génétiques & $\mathbf{4 7 , 8}$ & $\mathbf{2 8 , 6}$ & $\mathbf{1 9 , 9}$ & $\mathbf{6 2 , 3}$ & $\mathbf{2 2 , 0}$ & $\mathbf{1 2 , 0}$ \\
Pollution des lacs, rivières et mers & 42,4 & 36,4 & 20,1 & 49,9 & 29,2 & 20,2 \\
Produits végétaux génétiquement modifiés & 45,3 & 30,7 & $\mathbf{2 0 , 9}$ & $\mathbf{5 7 , 6}$ & $\mathbf{2 7 , 2}$ & $\mathbf{1 2 , 0}$ \\
Déchets radioactifs & 43,1 & 33,7 & 21,6 & 60,2 & 24,5 & 13,9 \\
Déchets chimiques & 38,1 & 37,6 & 22,0 & 55,4 & 29,3 & 13,7 \\
Installations chimiques & 35,2 & 38,0 & 23,1 & 52,6 & 30,3 & 13,3 \\
Pollution atmosphérique & 36,2 & 37,4 & 25,7 & 39,4 & 31,9 & 28,0 \\
Bruit & 37,9 & 34,0 & 26,5 & 29,9 & 32,5 & 35,5 \\
Produits alimentaires & 35,3 & 35,8 & 28,6 & 45,2 & 33,7 & 20,5 \\
Drogue & 38,0 & 32,3 & 29,0 & 32,8 & 19,1 & 47,7 \\
Centrales nucléaires & 36,7 & 32,7 & 29,0 & 56,8 & 26,1 & 16,1 \\
Tabagisme des jeunes & 40,2 & 28,6 & 30,3 & 23,8 & 24,7 & 50,5 \\
Transport des matières dangereuses & 33,1 & 34,4 & 31,6 & 49,2 & 29,3 & 20,0 \\
Radioactivité naturelle & 28,0 & 31,4 & 32,2 & 39,0 & 32,1 & 21,9 \\
Eau du robinet & 25,7 & 35,0 & 38,2 & 31,7 & 35,5 & 31,3 \\
Déchets ménagers & 22,8 & 37,7 & 38,9 & 30,4 & 36,7 & 31,1 \\
SIDA & 24,4 & 27,7 & 47,0 & 22,0 & 22,4 & 55,1 \\
Accidents de la route & 22,1 & 30,0 & 47,6 & 13,6 & 23,3 & 62,3 \\
\hline
\end{tabular}


goûts et les comportements d'achat des consommateurs grâce à l'exploitation de vastes banques de données par exemple. La référence à la qualité dans ses différentes acceptions est devenue omniprésente. Dans la recherche agronomique, la demande des consommateurs et, plus largement, la demande sociale sont également devenues un élément central pour I'orientation des recherches (Paillotin et Rousset 1999). Enfin les associations consuméristes sont maintenant représentées dans divers comités et les plus importantes sont de plus en plus courtisées. Cette réponse paraît a priori tout à fait adéquate et pertinente. Pourtant diverses questions peuvent se poser, en particulier pour la Recherche publique si ses orientations découlent trop directement de la demande sociétale ou des consommateurs même après divers relais. En effet la demande sociale exprimée peut parfois refléter des préoccupations catégorielles ou myopes comme l'a montré par exemple en 2000 la revendication d'une essence moins chère. Par ailleurs certains groupes sociaux ont bien plus de moyens pour faire entendre et prendre en compte leurs attentes, d'où de possibles biais. En outre comme la Recherche privée joue un rôle croissant, elle assure une part importante de la réponse à la demande, notamment dans l'alimentation pour ce qui concerne les aspects de qualité organoleptique. Dans ce contexte, la Recherche publique pourrait peut-être se positionner un peu différemment, au moins en partie : d'une part analyser les impacts sanitaires, écologiques et socio-économiques des évolutions techniques en cours, d'autre part explorer des voies technologiques (ou des composantes) un peu différentes, par ailleurs viser des approches prospectives, en tout cas de plus long terme, et enfin se soucier d'une meilleure prise en considération des besoins des populations peu solvables (souvent délaissees par les firmes) et de tout ce qui concerne les biens collectifs et les biens publics. Cette orientation existe déjà, mais devrait sans doute être corroborée.

II semble ainsi nécessaire de trouver une voie entre deux écueils :

- le refus d'entendre certaines demandes ou craintes des consommateurs en les taxant d'irrationnelles et en les condamnant, comme cela était le cas naguère pour les produits biologiques ou pour l'inquiétude face aux OGM. Ces deux exemples montrent que cette attitude peut parfois conduire à un renforcement ulterieur de ce qu'on a d'abord refusé énergiquement d'entendre!

- au contraire, la prise en considération trop littérale des demandes, pour relégitimer son activité aux yeux du grand public, sans suffisamment les relativiser, les hiérarchiser et surtout prendre un certain recul avec une vision à plus long terme.

\section{b / Une réponse surtout technique à une demande qui n'est pas seulement d'ordre technique}

Une réponse très fréquente pour réduire les risques sanitaires est l'instauration de nombreuses normes et réglementations en matièINRA Productions Ani males, décembre 2000 re d'hygiène. Or celles-ci peuvent comporter des effets pervers : par exemple, en cas de transformation et vente à la ferme de produits laitiers ou autres, la lourdeur des investissements requis pour satisfaire aux normes risque de ne les rendre accessibles qu'à de grandes exploitations, et donc de favoriser la concentration au lieu de permettre le maintien d'exploitations plus petites notamment en zones difficiles. La mise en place de la tracabilité génère aussi des contraintes assez lourdes. Il faut également pouvoir faire face à la concurrence provenant des pays aux normes moins rigoureuses. Aussi une analyse coûts-bénéfices d'une réglementation peutelle s'avérer fort utile avant de la mettre en place pour hiérarchiser les différentes mesures possibles (Arrow et al 1996).

Pour améliorer la qualité organoleptique des aliments, souvent décriée, et leur perception sensorielle, de très nombreux travaux fort sophistiqués sont conduits sur les multiples caractéristiques biophysicochimiques qui les influencent Or le rapport à la nourriture est le produit complexe de multiples influences historiques, géographiques, sociales, économiques, psychologiques ; de plus le goût ou la saveur perçus par les consommateurs ne résultent pas uniquement de leurs caractéristiques biophysicochimiques, mais aussi de nombreux autres aspects : cadre et contexte social et relationnel du repas, symbolique et image de l'aliment, etc (Giachetti 1997, Paillat 1997, Lahlou 1998, Gurviez 2000). La consommation alimentaire a un fort enracinement émotionnel, de ce fait une communication «technicienne» et rationnelle peut ne pas être entendue «ll y a un écart entre les réponses techni ci ennes de l'industrie agro-ali mentai re sur la gesti on du ri sque et les attentes des consommateurs qui concernent autant un apport de sens que d'énergi e» (Gurviez 2000). Autrement dit, mieux connaître l'image de l'agriculture moderne et de ses produits - en particulier de tout ce qui est souvent déprécié en tant qu' «alimentation industrielle»- serait aussi utile si I'on veut améliorer la saveur percue des aliments. Des actions sont certes menées pour mieux faire connaître l'agriculture et ses techniques et améliorer leur image, comme les rencontres citoyennes de l'alimentation en 2000, et l'accent mis sur l'agriculture raisonnée depuis quelques années (Bonny 1997a, Paillotin 2000). Mais suffisamment de recherches en sciences humaines et sociales ont-elles été conduites dans ces domaines et surtout prises en considération?

Les critiques d'une partie des consommateurs et des journalistes ne sont pas seulement d'ordre technique. Par delà les doléances «aliments insipides, gorgés d'hormones, d'antibiotiques, d'additifs, agriculture polluante...», il y a aussi des interrogations d'une autre nature, par exemple sur le sens de la quête incessante de la productivité malgré ses externalités négatives alors que beaucoup de secteurs connaissent des crises de surproduction. Figurent aussi parfois des questionnements sur l'instrumentalisation du monde vivant et son assujettissement à une vision productiviste utilitariste. Or, en fait, cette quête productiviste touche beaucoup de sec- 
teurs économiques et une cause essentielle en est le contexte de compétition économique exacerbée, non les techniques agricoles ou alimentaires à elles seules; mais la sensibilité des consommateurs est ici bien plus forte. Une des réponses fréquentes de l'industrie est celle de la différenciation verticale des produits avec le développement de divers labels et signes de qualité : le prix de vente plus élevé rend économiquement possible l'emploi des techniques plus traditionnelles ou plus extensives. Mais certains jugent choquant ce système dual où les uns peuvent s'offrir une nourriture labellisée «de qualité» alors que les autres doivent se contenter d'une nourriture industrielle perçue comme frelatée.

Quant aux pollutions agricoles, divers travaux ont été conduits pour les réduire, notamment par la recherche agronomique qui en a fait un objectif prioritaire depuis plusieurs années (Bonny 1993, INRA 1999). Mais les résultats ne sont pas tous mis en pratique, notamment parce que cela peut être plus difficile à mettre en œuvre et plus risqué au niveau économique (comme dans le cas de la production intégrée), alors que les incitations économiques sont en général faibles. En effet, hors du cas très spécifique de l'agriculture biologique, il n'existe quasiment pas d'écolabel en agriculture. Cependant diverses tentatives commencent à apparaître avec par exemple la promotion récente de «l'agriculture raisonnée». Mais si une part des consommateurs réclame des produits «de qualité», il ne faut pas oublier que le prix demeure primordial pour une partie des consommateurs.

De son côté, la demande de naturel, de naturalité, ne renvoie pas seulement à de seuls aspects techniques, mais aussi à des aspects symboliques. Le naturel et la naturalité reflètent en effet souvent la notion d'origine, de mère, de terre nourricière et, par là, quelque chose qui protège contre les angoisses relatives à l'avenir. Ce n'est donc pas la nature «réelle» qui est demandée (elle n'est pas spécialement bonne pour l'homme !). La demande de naturalité correspond en quelque sorte à une «recherche de sécurité affective, de besoin de savoir d'où proviennent nos aliments» (Hubert 2000). Cela correspond au besoin de lien entre nouveauté et origine, aux besoins de relations et d'échanges non destructifs pour permettre un développement plus harmonieux. A cette demande en partie d'ordre symbolique une réponse purement technique ne suffit sans doute pas.

\section{Conclusion et perspectives}

Ainsi, désormais, les interrogations en matière de qualité et de sûreté alimentaire sont fortement prises en considération et conduisent à une forte mobilisation de toute la filière : différenciation des produits permettant une variété de choix entre aliments courants et produits du terroir, sous label ou biologiques ; nombreux travaux fort pointus sur des critères de qualité ; édiction de nouvelles normes et réglementations sanitaires; mise au point de techniques plus sûres et plus propres ; etc. Cependant on peut se demander si tous ces efforts pour la sûreté alimentaire, pour l'amélioration de différents critères de qualité, notamment organoleptiques, seront suffisants. Les consommateurs expriment en effet souvent par le terme de «malbouffe» leur perception de l'emploi de matières premières peu nobles. Or, en ce domaine, I'une des causes principales est le contexte de compétition économique exacerbée qui paraît difficile à contourner, même avec la différenciation verticale des produits.

Par ailleurs les interrogations et les doutes sur la sûreté sanitaire peuvent cristalliser des inquiétudes plus profondes comme le montre le cas des OGM : inquiétudes par rapport à l'évolution du système économique global (concentration des firmes, mondialisation, concurrence exacerbée), la critique technique exprimerait aussi une critique plus socio-économique ; inquiétudes par rapport à la complexification et l'évolution techniques et économiques très rapides perçues comme une fuite en avant (B onny 2000b). Dans ce contexte, I'agriculture traditionnelle est ressentie comme équilibrée et harmonieuse et l'agriculture moderne au contraire comme «folle» (selon certaines dénonciations) et détruisant les équilibres naturels là où elle s'étend. Même si la réalité est bien plus complexe que ces images d'Epinal, on ne peut balayer ces perceptions sans en tenir compte. Aussi n'estil pas sûr que les réponses apportées ou recherchées aujourd'hui face aux critiques des consommateurs s'avèrent suffisantes car l'inquiétude des consommateurs en matière d'alimentation pourrait renvoyer aussi à une certaine crise de confiance envers le système économique «technocratique». En effet l'individualisation croissante peut entraîner un sentiment d'insécurité en raison de la diminution du lien social et des référents identitaires. D'où l'apparition de besoins de réassurance, se traduisant notamment sur le plan de la consommation alimentaire par la montée des thèmes comme le terroir, la nature, l'environnement, la santé, la solidarité, accompagnés d'une demande croissante de sens, de transparence et d'éthique (Gurviez 2000).

Face aux divers facteurs de remise en question, l'une des réponses pourrait être une évolution vers un nouveau type d'agriculture, par exemple une agriculture diversifiée, plurielle et multifonctionnelle, susceptible ainsi d'une meilleure adaptation à la diversité des contextes, des contraintes et des demandes, et qui viserait également une plus grande durabilité (Bonny 1997b). Mais une telle évolution sera-t-elle possible alors que les forces économiques conduisent souvent les agriculteurs à rechercher l'agrandissement de leurs exploitations et entraînent également une forte chute du nombre des installations ? La demande de sécurité sanitaire et même d'environnement, peut certainement être intégrée techniquement par le système économique : mais la dynamique d'évolution vers l'industrialisation de l'agriculture, et la concentration des exploitations au niveau local et celle des grandes firmes au niveau planétaire seront-elles freinées pour autant?

INRA Producti ons Ani males, décembre 2000 


\section{Signification des sigles}

BVA, CSA, IFOP, IPSOS, ISL, SOFRES sont des instituts de sondages.

ANIA : Association Nationale des Industries Alimentaires CE : Commission Européenne

CREDOC : Centre de Recherche pour l'Etude et I'Observation des Conditions de Vie

DG : Direction Générale (à la CE)

ESB : Encéphalopathie Spongiforme Bovine

FARRE : Forum de l'agriculture raisonnée respectueuse de l'environnement

INED : Institut National d'Etudes Démographiques

INSEE : Institut National de la Statistique et des Etudes

Economiques

INSERM : Institut National de la Santé et Recherche Médicale

IPSN : Institut de Protection et de Sûreté Nucléaire

ISL : Institut de Sondage Lavialle
LSA : Libre - Service-Actualités (magazine de la Distribution) OGM : Organisme Génétiquement Modifié

PAC : Politique agricole commune

SIGMA-UNCAA : Union de coopératives agricoles céréalières européennes

SNIA : Syndicat National des Industriels de la nutrition Animale SOFRES : Société FRançaise d'E nquête par Sondage SYNCOPAC : Syndicat national des coopératives de production et d'alimentation animales

UFC : Union fédérale des consommateurs

UIPP : Union des Industries de la Protection des Plantes.

\section{Remerciements}

L'auteur remercie bien sincèrement les divers organismes et les instituts de sondage qui lui ont fourni les résultats des enquêtes qu'ils avaient réalisées.

\section{Références}

Apfelbaum M. (ed), 1998. Risques et peurs alimentaires. E ditions Odile J acob, Paris, 244 p.

Arrow K.J. et al, 1996. Is there a role for benefitcost analysis in environment, health, and safety regulation? Science, 272, 221-222

Bayle N., Malait M.J., Laperrousaz P., 1999. La peur des OGM et les intérêts des industriels. Problèmes économiques, 2628, 25-29.

Bonny S., 1993. Le changement technique en cours et à venir en agriculture : un essai de bilan dans les différents secteurs. Grignon, INRA ESR, Etudes économiques $n^{\circ} 12,134 p$

Bonny S., 1997a. L'agriculture raisonnée, l'agriculture intégrée et l'association FARRE (forum de I'agriculture raisonnée respectueuse de l'environnement). Natures-Sciences-Sociétés ,5, 64-71.

Bonny S., 1997b. Quelles perspectives d'évolution possible pour l'agriculture francaise face aux mutations en cours ? Norois (Revue géographique de l'Ouest et des pays de l'Atlantique Nord), numéro spécial «crises et mutations agricoles et rurales» (44) 173, janvier-mars, 121-140.

Bonny S., 2000a. Consumer concerns about industrialized agriculture and food safety: importance, origin and possible solutions. Annales de Zootechnie, 49, 273-290.

Bonny S., 2000b. Why are most Europeans opposed to GMOs? Explanatory factors underlying the rejection movement: the French case. 24th International Conference of Agricultural Economists (IAAE), Berlin, 13-18 August 2000: «Tomorrow's Agriculture: Incentives, Institutions, Infrastructure and Innovations».

Boy D., 1999. Le progrès en procès. Presses de la Renaissance, Paris, $265 \mathrm{p}$.

(The) BSE Inquiry report, 2000. The inquiry into BSE and variant CJ D in the UK, by Lord Phillips of Worth Matravers et al. Consultable sur le site <http://www.bseinquiry.gov.uk/index.htm>
Cazes-Valette G., 1998. Anthropologie et comportement du consommateur : le cas de la vache folle. In: Fisler M. Lemoine J.F. (eds), Comportement du consommateur, Actes de la Zéme journée de Recherche en Marketing de Bourgogne, Dijon, Université de Bourgogne, Centre de Recherche en Economie et Gestion des Organisations, 4 juin 1998, 4-22.

CE, 1997. Les européens et la Biotechnologie moderne. Eurobaromètre 46.1. CE, DG XII Science, Recherche et developpement, Bruxelles.

CE, 1998. La sécurité des produits alimentaires. Eurobaromètre 49. CE, DG XXIV Consumer Policy, Bruxelles.

CE, 1999. Standard Eurobarometer Public opinion in the European Union. Report Number 50. CE, DG $X$, Bruxelles.

CE, 2000. Les Européens et la biotechnologie. Eurobaromètre 52.1. CE Direction Générale Recherche et Direction générale de l'E ducation et de la Culture, Bruxelles, 84 p + rapports annexes.

Chateauraynaud F., Torny D., 1999. Les sombres précurseurs. Une sociologie pragmatique de l'alerte et du risque. Editions de l'EHESS (Ecole des hautes études en sciences sociales), Paris, $476 \mathrm{p}$.

CREDOC, 1998. Les opinions des français sur la qualité et sur les risques sanitaires des produits alimentaires. CRE DOC, Collection des rapports, 190, Paris.

Espey J., 1998. Socioethical implications of biotechnology. Industry Canada, Office of Consumer Affairs, Ottawa, Canada.

Feillet P. (ed), 1998. Aliments et industries alimentaires : les priorités de la recherche publique. INRA, Paris, $288 \mathrm{p}$.

Fischler C., 1993. L'Homnivore, 2ème édition. Editions Odile J acob, Paris, $440 \mathrm{p}$

Gaskell G., Bauer M.W., Durant J ., Allum N.C., 1999 Worlds Apart? The Reception of Genetically Modified Foods in Europe and the U.S. Science, 285, 384-387. 
Gaskell G. et al, 2000. Biotechnology and the European public. Nature Biotechnology, 16, September 2000, 935-938.

Giachetti I. (ed.), 1997. Identités des mangeurs, images des aliments. Editions Polytechnica, Paris, $217 \mathrm{p}$.

Gilbert C., 1998. Le sens caché des risques collectifs. La Recherche, 307, 110-113.

Gurviez P., 2000. Consommation alimentaire et confiance du consommateur. Actes des lers Ateliers de recherche sur le risque en marketing, 9 juin 2000. Paris, Université Panthéon-Sorbonne, Laboratoire de stratégie industrielle, 197-213.

Hornig Priest S., 2000. US public opinion divided over biotechnology? Nature Biotechnology, 18, September 2000, 939-942

Hubert A., 2000. Alimentation et santé : la science et l'imaginaire. Cahiers de Nutrition et de Diététique, 35, 5, 353-356.

INRA., 1999. L'INRA, Recherche et Innovation Alimentation, environnement, agriculture et société (plaquette de présentation des nouvelles orientations de I'INRA). INRA, Paris, $25 \mathrm{p}$.

IPSN (Institut de protection et de sûreté nucléaire), 2000. Perception des risques et de la sécurité. Résultats du sondage d'octobre 1999. Baromètre IPSN. Clamart, 2000

Lahlou S., 1998. Penser manger. Alimentation et représentations sociales. Presses Universitaires de France, Paris, $256 \mathrm{p}$.

Loder N., 1999. BSE advisers admit giving up a purely scientific role. Nature, $500,490$.

Miclet G., Sirieix L., Thoyer S. (eds), 1998. Agriculture et alimentation en quête de nouvelles légitimités. Editions E conomica, Paris, 371 p.

Morgan G., 1993. Risk analysis and management. Scientific American, 269, 32-41 (en français: L'évaluation et la gestion des risques, Pour la Science, numéro spécial: Le hasard, avril 1996, 120-126).
Nizard A., 1999. Du SIDA et d'autres risques émergents. Population et Sociétés, 349, sept 1999.

Paillat M. (ed), 1997. Le mangeur et I'animal. Mutation de l'élevage et de la consommation. E ditions Autrement, Paris, 151 p.

Paillotin G., 2000. L'agriculture raisonnée. Rapport au ministre de l'agriculture. INRA, Paris, $53 \mathrm{p}$.

Paillotin G., Rousset D., 1999. Tais-toi et mange! L'agriculteur, le scientifique et le consommateur. Editions Bayard Centurion, Paris, 182 p.

Paillotin G., Landais E., Savini I., 1999. Les nouvelles responsabilités des scientifiques face aux incertitudes du Futur. Cahiers Agricultures, 8.

Powell D., 1998. Impacts of biotechnology, environment, food safety: communications. Paper for the Agriculture Risk Management Conference held at Hull, Canada. University of Guelph, Canada.

Slovic P., 1987. Perception of risk. Science, 236, 180-285.

Slovic P. et al, 1995. Intuitive toxicoloy. II. Expert and lay judgments of chemical risks in Canada. Risk Analysis, 15, 661-675.

Tubiana M., 1998. Alimentation et cancer. Bulletin de l'Académie Nationale de Médecine, 182, 18871897.

Tubiana M., Vrousos C., Carde C., Pagès J.P. (eds), 1999. Risque et société. E ditions Nucléon, Gif-surYvette, $459 \mathrm{p}$.

Valceschini E., 1999. Pertinence et faisabilité d'une filière «sans OGM». Paris, INRA et FNSEA, rapport intermédiaire, nov. 1999, 24 p.

Viel J.M., 1979. L'agriculture biologique : une réponse? Editions Entente, Paris, $93 \mathrm{p}$.

Zechendorf B., 1998. Agricultural biotechnology: why do Europeans have difficulty accepting it? AgBioF orum, 1, 8-13.

\section{Abstract}

Consumers, agriculture, food quality and food safety : an analysis of consumer questions and of the answers put forward.

This article analyses the worries and the criticisms concerning modern agriculture and food often expressed today on the one hand, and, on the other, the answers put forward by the actors concerned. First, the consumer risk perceptions of food quality and of food safety are studied using the results from numerous recent surveys and polls. The article then presents some underlying explanatory factors motivating consu- mer questions about current agriculture and food. On this basis, the appropriateness of the answers proposed to the risks denounced and to this confidence crisis is examinated. In fact, the answers often appear to be relatively technical, whereas the points of concern are not limited to technical matters.

BONNY S., 2000. Les consommateurs, l'agriculture, la qualité et la sécurité des aliments : une analyse du questionnement des consommateurs et des réponses apportées. INRA Prod. Anim., 13, 287-301.

I NRA Producti ons Ani males, décembre 2000 
